\title{
A SIXTH ORDER INVERSE POTENTIAL FUNCTION FOR INCORPORATION OF CRYSTALLOGRAPHIC TEXTURE INTO PREDICTIONS OF PROPERTIES OF ALUMINIUM SHEET
}

\author{
JEAN SAVOIE and STUART R. MacEWEN \\ Alcan International Limited, Kingston Research and Development Center, \\ P.O. Box 8400, Kingston (Ontario), Canada
}

(Received 28 December 1995)

\begin{abstract}
In order to simulate adequately the formability of sheet, it is necessary to generate a yield criterion that takes into account the anisotropy of the crystallographic texture. This yield function must be in a form that can be used easily in finite element analysis. In addition, the formulation should allow the possibility to update the yield surface during simulation to take into account the texture evolution. This work investigates the use of a sixth-order strain rate potential that, when differentiated with respect to the strain rate, gives the components of stress. A method is given to solve the non-convexity problems that can arise when the proposed analytical sixth-order function is used. Examples are given for one cold rolled and one annealed aluminium sheet.
\end{abstract}

KEY WORDS: Inverse potential, yield surface, texture, anisotropy

\section{INTRODUCTION}

The anisotropy of mechanical properties of rolled sheet, and thus directly its ability to be formed into consumer products such as car panels and beverage cans, is controlled to a large extent by the preferred orientations, or texture, of the material. In order to simulate mathematically the formability of sheets, it is necessary to capture the information carried by the texture in an efficient, mathematical form that can be used in a variety of analysis packages, but primarily in finite element (FE) analysis.

The fundamental entity linking texture to properties is the yield surface, which defines the boundary between elastic and plastic response for a multi-axial stress state. The normal to the yield surface defines the strain rate response to the imposed stress state. Finite element simulations of metal forming are commonly performed using the Von Mises definition of the yield surface and effective strain. However, such representation neglects completely the anisotropy introduced due to crystallographic texture. In order to reproduce faithfully this anisotropy, the definition of the yield surface must include the effects of texture. In addition, since texture evolves during deformation, the formulation of the yield surface must be able to be updated rapidly during an analysis.

Three types of procedures have been used previously in the literature [see a review of these procedures in Bacroix and Gillormini (1995)]. The first, and most common approach is to represent the yield surface by a closed-form, analytical expression of 
the five independent components of stress. The isotropic, quadratic Von Mises equation is the most simple. Anisotropy has been included into the quadratic Hill formulation [Hill (1948)] and by defining non-quadratic functions [for example, Hill (1979), Barlat (1987), Hill (1990)]. In principle, the constants of the equations can be determined by a series of experiments to define yield stress and $\mathrm{R}$-values in specific directions of the sample. In practice, the experiments can be difficult and time consuming. The principal advantage of a simple, analytical function is that it can be easily incorporated into a FE analysis. The disadvantages are that the shape of the function may be only a poor approximation to reality and that there is no option for updating the shape during a simulation, as the texture evolves.

The second approach involves the use of the basic concepts of crystal plasticity, which are well established. Several authors, notably Kocks and Canova (1981), Van Houtte (1988), Asaro and Needleman (1985), and Toth et al. (1988) have developed standalone models, based on slip plane activity. These models are, in a sense, equivalent to a one-element finite element model. Incorporation of crystal plasticity directly into $\mathrm{FE}$ analysis as the "material" subroutine has been achieved successfully by several authors, notably Mathur and Dawson (1989), but all suffer from the immense amount of computation power and hardware resources required for execution. The most successful application is that by Beaudoin et al. (1994) who used a massively parallel platform to simulate sheet forming operations using crystal plasticity.

The third approach combines the best of the first two by fitting (in a least-squares sense) the constants of a simple analytical function to "data" produced by crystal plasticity calculations, rather than by experimentation. Two options exist: to use crystal plasticity calculations of the yield surface or crystal plasticity calculations of the plastic work rate for the regression fitting exercise. The latter defines a function of the strain rate components (rather than the stress components). Fitting to the yield surface has been already accomplished in the five components of stress, Barlat and Lian (1989). The alternative is to fit the analytical function not to a yield surface but to the inverse potential, a function that when differentiated with respect to strain rate gives the components of stress, Van Houtte et al. (1989), Arminjon and Bacroix (1991), Barlat (1993). The regression analysis of the inverse potential function is easier and can be introduced directly into FE codes, Bacroix and Gillormini (1995). The fitting procedure proposed by Arminjon and Bacroix (1991) has the notable advantage to be performed only once on functions that are independent of any texture. The update of yield surfaces during deformation, which can be necessary due to texture evolution, is then obtained simply and rapidly. A fourth-order strain rate potential function introduced by Arminjon et al. (1994) and tested successfully for steels, could not reproduce the sharp "corners" observed on the yield surfaces of aluminium alloys. For this reason, in this work, the use of a sixth-order function, already introduced by Bacroix and Gillormini (1995), is investigated.

\section{DEFINITION OF THE STRAIN RATE OR INVERSE POTENTIAL}

The concept of strain rate potential, or inverse potential, was introduced by Ziegler in 1977 and Hill in 1987. Since then several authors, notably Van Houtte et al. (1989), Arminjon and Bacroix (1991) have built on the basic concept, which is outlined below.

The yield criterion for a textured polycrystal (yield surface) can be represented by:

$$
\mathrm{F}(\sigma)=\tau
$$


and the associated flow rule, which defines the strain rate response for a given stress state by:

$$
\mathrm{D}_{\mathrm{i}}=\dot{\lambda} \frac{\partial \mathrm{F}}{\partial \sigma_{\mathrm{i}}}
$$

The inverse potential reverses the roles of stress and strain rate and defines a function of the strain rate that can be differentiated to give the stress state. Thus the inverse, or dual potential is written as:

$$
\psi(\mathbf{D})=\dot{\lambda}
$$

and its associated flow rule becomes:

$$
\sigma_{\mathrm{i}}=\tau \frac{\partial \psi}{\partial \mathrm{D}_{\mathrm{i}}}
$$

The parameters $\tau$ and $\dot{\lambda}$ are scalar variables. If the $\psi$ function is homogeneous of degree one, the dual potential becomes, simply:

$$
\psi(\mathbf{D})=\frac{\dot{\mathrm{W}}(\mathbf{D})}{\tau}
$$

The strain rate potential is therefore nothing else than the stress-normalized plastic work rate.

\section{ANALYTICAL YIELD SURFACE CALCULATION}

The discrete values of the crystallographic dual potential, $\psi_{\mathrm{C}}(\mathbf{D})$, defined in terms of the plastic work rates, $\dot{\mathrm{W}}_{c}(\mathbf{D})$, calculated from a crystal plasticity code and a given texture, can be represented by a simple function, homogeneous of degree one, $\psi(\mathbf{D})$, that can be incorporated into finite element analysis without the vast overhead associated with incorporation of crystal plasticity directly. According to Arminjon and Bacroix (1991), such a function can be expressed as,

$$
\psi(\mathbf{D})=\sum_{\mathrm{k}=1}^{N} \alpha_{k} X_{k}(\mathbf{D})
$$

This dual potential function consists of $k$ terms, $X_{k}$, each with a multiplier, $\alpha_{k}$. These multipliers, or anisotropy coefficients, must be determined by fitting, in a least-squares regression sense, to the "data" provided by the crystal plasticity analysis, using an appropriate sampling of plastic strain rate tensors D. Thus:

$$
\sum_{\mathbf{D}}\left\{\psi_{c}(\mathbf{D})-\psi(\mathbf{D})\right\}^{2}=\sum_{\mathbf{D}}\left\{\frac{\dot{\mathbf{W}}_{c}(\mathbf{D})}{\tau}-\psi(\mathbf{D})\right\}^{2}=\min .
$$

Using the series expansion formalism, one obtains:

$$
\sum_{\mathbf{D}}\left\{\sum_{\ell \mu \mathrm{n}} \frac{\mathrm{C}_{\ell}^{\mu \mathrm{u}} \cdot \dot{\mathrm{w}}_{\ell}^{\mu \mathrm{n}}(\mathbf{D})}{2 \ell+1}-\sum_{\mathrm{k}} \alpha_{\mathrm{k}} \mathrm{X}_{\mathrm{k}}(\mathbf{D})\right\}^{2}=\min
$$

where $\mathrm{C}_{\ell}^{\mu n}$ and $\dot{\mathrm{w}}_{\ell}^{\mu n}$ are the texture and plastic work rate coefficients, respectively. However, according to Arminjon and Bacroix (1991), if the analytical function is a 
polynomial of the form of Equation (4), it is advantageous to separate the texture coefficients from the regression and minimize the residual of:

$$
\sum_{\mathbf{D}}\left\{\dot{\mathrm{w}}_{\ell}^{\mu \mathrm{n}}(\mathbf{D})-\sum_{\mathrm{k}} \beta_{\ell \mathrm{k}}^{\mu \mathrm{n}} \cdot \mathbf{X}_{\mathrm{k}}(\mathbf{D})\right\}^{2}=\min \text {. }
$$

to find the $\beta$ 's rather than the $\alpha$ 's. The fitting procedure is thus independent of texture. It is only a function of the deformation model used to calculate the plastic work rate coefficients $\dot{w}_{\ell}^{\mu n}$. In this way, the lengthy regression analysis to find the $\beta$ 's needs be done only once. The anisotropy coefficients of any particular texture can be found as follows:

$$
\alpha_{\mathrm{k}}=\sum_{\ell \mu \mathrm{n}} \frac{\mathrm{C}_{\ell}^{\mu \mathrm{n}} \cdot \beta_{\ell \mathrm{k}}^{\mu \mathrm{n}}}{2 \ell+1}
$$

The degree of expansion $\ell$ that must be used depends on the severity of the texture considered. For the textures investigated (one cold rolled can body stock and one recrystallized automotive material), a series expansion degree $\ell_{\max }=22$ is sufficient to represent the orientation distribution function. However it is not necessary to reach $\ell_{\max }=22$ in order to define the dual potential, $\dot{\mathrm{W}}_{\mathrm{c}}(\mathbf{D}) / \tau$, as the plastic work rate coefficients define a smooth function (the values of the $\beta$ 's decrease rapidly as $\ell$ increases). For this reason, the degree of expansion was set to $\ell_{\max }=12$ without changing the accuracy of the potential for the textures considered. This choice reduces considerably the calculation time as only 38 expansion terms $(\ell \mu \mathrm{n})$ are used, instead of 171 for $\ell_{\max }=22$.

\section{Form of the dual potential $\psi(D)$}

For describing the anisotropy of a sheet, Arminjon et al. (1994) proposed a dual potential function in the form of a fourth-order criterion, homogeneous of degree one:

$$
\psi(\mathbf{D})=\sum_{\mathrm{ijkl}} \alpha_{\mathrm{ijkl}} \frac{\mathrm{D}_{\mathrm{i}} \mathrm{D}_{\mathrm{j}} \mathrm{D}_{\mathrm{k}} \mathrm{D}_{1}}{\|\mathbf{D}\|^{3}}=\sum_{\mathrm{k}} \alpha_{\mathrm{k}} \frac{\mathrm{X}_{\mathrm{k}}}{\|\mathbf{D}\|^{3}}
$$

where $\mathrm{D}_{\mathrm{i}}$ is the component $i$ of the plastic strain rate tensor $\mathbf{D}$. The norm of $\mathbf{D}$ is defined as:

$$
\|\mathbf{D}\|=\sqrt{D_{i j}^{2}}=\sqrt{2} \sqrt{D_{11}^{2}+D_{22}^{2}+D_{11} D_{22}+D_{23}^{2}+D_{31}^{2}+D_{12}^{2}}
$$

This approach proved successful and the analysis described reasonably well the plasticity of steel, but did not represent the nature of the yield surface of aluminium sheet adequately. In particular, the long flat regions near plane strain and the sharp "corners" at equibiaxial and uniaxial positions were not represented adequately. In order the improve the representation, this work investigates the use of a sixth-order function, of the same general form as the fourth-order one introduced above:

$$
\psi(\mathbf{D})=\sum_{i j k l m n} \alpha_{i j k l m n} \frac{D_{i} D_{j} D_{k} D_{l} D_{m} D_{n}}{\|\mathbf{D}\|^{5}}=\sum_{k} \alpha_{k} \frac{X_{k}}{\|\mathbf{D}\|^{5}}
$$

Five independent components for $\mathbf{D}$ are used (namely: $\mathrm{D}_{11}, \mathrm{D}_{22}, \mathrm{D}_{23}, \mathrm{D}_{31}, \mathrm{D}_{12}$ ), since it is assumed that $D_{11}+D_{22}+D_{33}=0$. As an example, for the plane stress case with orthorhombic sample symmetry, the inverse potential function is composed of $16 \mathrm{X}_{\mathrm{k}}$ terms: 


$$
\begin{aligned}
& X_{1}=D_{11}{ }^{6} \\
& X_{2}=D_{11}{ }^{5} \cdot D_{22}{ }^{2} \\
& X_{3}=D_{11}{ }^{4} \cdot D_{22}{ }^{2} \\
& X_{4}=D_{11}{ }^{3} \cdot D_{22}{ }^{3} \\
& X_{5}=D_{11}{ }^{2} \cdot D_{22}{ }^{4} \\
& X_{6}=D_{11} \cdot D_{22}{ }^{4} \\
& X_{7}=D_{22}{ }^{6} \\
& X_{8}=D_{12}{ }^{2} \cdot D_{11}{ }^{4}
\end{aligned}
$$

$$
\begin{aligned}
& \mathrm{X}_{9}=\mathrm{D}_{12}{ }^{2} \cdot \mathrm{D}_{11}{ }^{3} \cdot \mathrm{D}_{22}{ }^{2} \\
& \mathrm{X}_{10}=\mathrm{D}_{12}{ }^{2} \cdot \mathrm{D}_{11}{ }^{2} \cdot \mathrm{D}_{22}{ }^{2} \\
& \mathrm{X}_{11}=\mathrm{D}_{12}{ }^{2} \cdot \mathrm{D}_{11} \cdot \mathrm{D}_{22}{ }^{3} \\
& \mathrm{X}_{12}=\mathrm{D}_{12}{ }^{2} \cdot \mathrm{D}_{22}{ }^{4} \\
& \mathbf{X}_{13}=\mathrm{D}_{12}{ }^{4} \cdot \mathrm{D}_{11}{ }^{2} \\
& \mathrm{X}_{14}=\mathrm{D}_{12}{ }^{4} \cdot \mathrm{D}_{11} \cdot \mathrm{D}_{22} \\
& \mathrm{X}_{15}=\mathrm{D}_{12}{ }^{4} \cdot \mathrm{D}_{22}{ }^{2} \\
& \mathrm{X}_{16}=\mathrm{D}_{12}{ }^{6}
\end{aligned}
$$

For the general function in five dimensional strain-rate space, with orthorhombic and triclinic sample symmetries, there are 60 and $210 \mathrm{X}_{\mathrm{k}}$ terms, respectively. The number of anisotropy coefficients as a function of the order of the polynomial function are listed in Table 1.

Table 1 Number of anisotropy coefficients as function of the order of the function.

\begin{tabular}{lcrc}
\hline & $\begin{array}{c}\text { Orthorhombic } \\
\text { Plane stress }\end{array}$ & $5 D$ & $\begin{array}{c}\text { Triclinic } \\
5 D\end{array}$ \\
\hline 2nd order & 4 & 5 & 25 \\
4th order & 9 & 22 & 70 \\
6h order & 16 & 60 & 210 \\
8th order & 25 & 135 & 490 \\
\hline
\end{tabular}

The orthorhombic symmetry can be used for most applications when the initial texture is orthorhombic (for example, rolling sheet whose texture axis corresponds to the sample axis). For other cases (i.e. non orthorhombic texture and for texture updating during the simulation), all the coefficients for triclinic symmetry must be used. From this table, it can be seen that the number of coefficients is considerably reduced if a plane stress state (instead of a general 5D state) is considered.

Once the analytical expression of the strain rate potential is determined, the stress associated with a given plastic strain rate is derived from the associated normality rule:

$$
\sigma_{\mathrm{i}}=\tau_{\mathrm{c}} \frac{\partial \psi(\mathbf{D})}{\partial \mathrm{D}_{\mathrm{i}}}
$$

Because the macroscopic strain rate potential has been fitted to the crystallographic one, the reference stress used in the normality rule is thus $\tau_{c}$, the critical shear stress of the slip systems, not a yield stress measured in tension.

\section{Fitting procedure}

The "data" for the regression analysis were generated using both rate insensitive and rate sensitive crystal plasticity with full constraints. Relaxed constrain models are not appropriate for this type of analysis (general 5D calculation), as they would generate physically unrealistic macroscopic shear terms which would be in conflict with the macroscopic conditions of the imposed strain rate tensor. Generation of the data requires sampling all of strain rate space (all possible $D_{i j}$ 's) for each possible crystallite 
orientation. These orientations must be selected in such a way that they cover the entire orientation space (all possible Euler angles) with appropriate symmetry.

Arminjon and Bacroix (1991) proposed a sampling of uniformly distributed $\mathrm{D}_{\mathrm{ij}}$ 's in the 5D strain rate space. The advantage of this method consist in the use of only few strain rate components. However, this method results in slightly more pronounced nonconvexity problems (see below). A non-uniform distribution of $D_{i j}$ 's is instead used, which involves a larger amount of sampling points.

In the case of the full constraint model, the crystal plasticity code needs to be run only using the strain rate tensors in principal axis (p). This particular tensor is defined with the contraction ratio $\mathrm{q}(0 \leq \mathrm{q} \leq 0.5$ for the $5 \mathrm{D}$ calculation), and takes the following form:

$$
\dot{\mathrm{E}}^{\mathrm{P}}(\mathrm{q})=\left(\begin{array}{ccc}
1 & 0 & 0 \\
0 & -\mathrm{q} & 0 \\
0 & 0 & -1+\mathrm{q}
\end{array}\right)
$$

Once obtained, the plastic work rate coefficients can be rapidly transformed from the principal axis into the sample axis to cover the whole strain rate space:

$$
\dot{\mathrm{w}}_{\ell}^{\mu \mathrm{n}}(\mathbf{D})=\dot{\mathrm{w}}_{\ell}^{\mu \mathrm{nn}}(\mathrm{q})\left(\theta_{1}, \theta, \theta_{2}\right)=\sum_{\mathrm{s}=-\ell}^{+\ell} \dot{\mathrm{w}}_{\ell}^{\mu \mathrm{s}^{\mathrm{P}}}(\mathrm{q}) \cdot \ddot{\mathrm{T}}_{\ell}^{\mathrm{ns}}\left[\mathrm{g}^{\prime}\left(\theta_{1}, \theta, \theta_{2}\right)\right]
$$

where $\ddot{T}_{\ell}^{\text {ns }}$ are the unsymmetrized spherical harmonic functions for a rotation $g^{\prime}\left(\theta_{1} \theta, \theta_{2}\right)$ defining the transformation. $\mathrm{g}^{\prime}$ has the following form:

$$
0^{\circ} \leq \theta_{1} \leq 360^{\circ} ; 0^{\circ} \leq \theta \leq 90^{\circ} ; 0^{\circ} \leq \theta_{2} \leq 180^{\circ}
$$

Several calculations were carried out using different resolutions for the fitting procedure. A first calculation involved 77,330 fitting points or strain rates, namely, 11 contraction ratio values $\mathrm{q}(\mathrm{q}=0,0.05,0.10,0.15, \ldots, 0.5$ with $\Delta \mathrm{q}=0.05)$ and 7030 rotation angles $\left(\theta_{1}, \theta, \theta_{2}\right)$ with $\Delta \theta=10^{\circ}$. Higher resolutions were used in order to investigate the quality of the fit with the number of sampling points up to $1,195,152,231$ fitting points $\left(\Delta q=0.0025\right.$ and $\left.\Delta \theta=1^{\circ}\right)$. It has been found that a sampling of more than 564,509 strain rates $\left(\Delta \mathrm{q}=0.05\right.$ and $\left.\Delta \theta=5^{\circ}\right)$ did not improve the fitting procedure. For this reason, only the latter resolution is used.

\section{SIMULATION RESULTS}

In this section, comparisons are made between the quality of the fit (for selected textures) of the fourth-order (Quartus) and sixth-order (Sextus) analytical functions. A sharp simulated single-component texture is first used to show the limitations of the method (appearance of non-convexity problems). Solutions are then proposed to solve these non-convexity problems. Then to test the quality of the fit (yield surface, yield strength and R-values), one cold rolled can body stock and one recrystallized automotive material are employed.

\section{(i) The non-convexity problem}

First calculations were carried out for a single component texture, as it was expected that the greatest non-convexity problems would then occur. For very sharp textures, 
the regression procedure produces a multi-valued function called "fish-tails", due to their appearance when plotted in 2D space. Figures $1 \mathrm{a}$ and $1 \mathrm{~b}$ show the analytical yield function and the analytical dual potential, respectively, for the ideal texture component $\{011\}<211>$ (Brass) with a Guassian spread of $9^{\circ}$ about its maximum. Each analytical function is compared with the corresponding curve obtained using the crystallographic (or "exact") approach. Figure $1 \mathrm{~b}$ displays the isopotential of the dual function and not the function $\psi(D)$ itself. It is convenient to remember that the yield surface is the representation of isopotential of $\sigma_{i}$, so that the function $F(\sigma)$ is constant (here equal to $\tau$ ). Conversely, the dual potential is the representation of $D_{i}$, so that the function $\psi(D)$ is constant (here equal to $\dot{\lambda}$ ). Therefore, in the later case, the Figure $1 \mathrm{~b}$ represents $\mathrm{D}_{\mathrm{i}} / \psi(\mathbf{D})$.

The analytical yield surface shows large "fish tails" at both extremities, and small ones at two other corners, which correspond to concave parts in the dual potential. The shape of the yield surface is directly connected to the shape of the strain rate potential. Concave, straight or convex parts in the dual potential will produce fish tails, points (discontinuities or ambiguities in the derivative) or convex part in the plastic potential, respectively. This lack of convexity, which is typically observed when high order function (6th and 8th order) are used ${ }^{1}$, is physically unacceptable and could lead to convergence problems if implemented into a finite element analysis.

\section{(ii) Approaches to solve the non-convexity problem}

Three different approaches have been investigated to solve the non-convexity problems: a) increasing the number of the fitting points during the least-squares procedure, b) minimization by Monte-Carlo search and c) the use of rate sensitive plastic work rates.

The first and most obvious one consists to increase the precision of the least-squares procedure by increasing the number of fitting points. However, as already pointed out in the fitting procedure section, this solution does not improve significantly the calculation.

The second method consists in two steps. The first performs a minimization by MonteCarlo search. Each time a new minimum is found, the search area is shifted to be centered at the best value. The last step involves a local function minimization using the method of Davidon-Fletcher-Powell as modified by Fletcher (1970). The use of this second method increases the quality of the fit by decreasing slightly the size of the fish tails obtained in a rate insensitive yield surface, but does not necessarily remove them.

The last method involves the use of rate sensitive plastic work rate coefficients (instead of rate insensitive ones). This has the effect of smoothening the sharp rate insensitive corners, as well as rounding the straight lines obtained in the dual potential plot. The analytical function tends then to be convex. Depending on the strength of the texture, different rate sensitivity exponents, $m$, have to be employed. Typically, $m=0.05$ is required for textures of medium strength, such as for the present can body stock material, see Figures 2 and 3. The analytical yield surface of the weaker texture observed in the automotive sheet in convex even for very low $m$ values ( $m \leq 0.01$ or rate insensitive).

'Fish tails are seldom observed when the fourth-order function is used. 


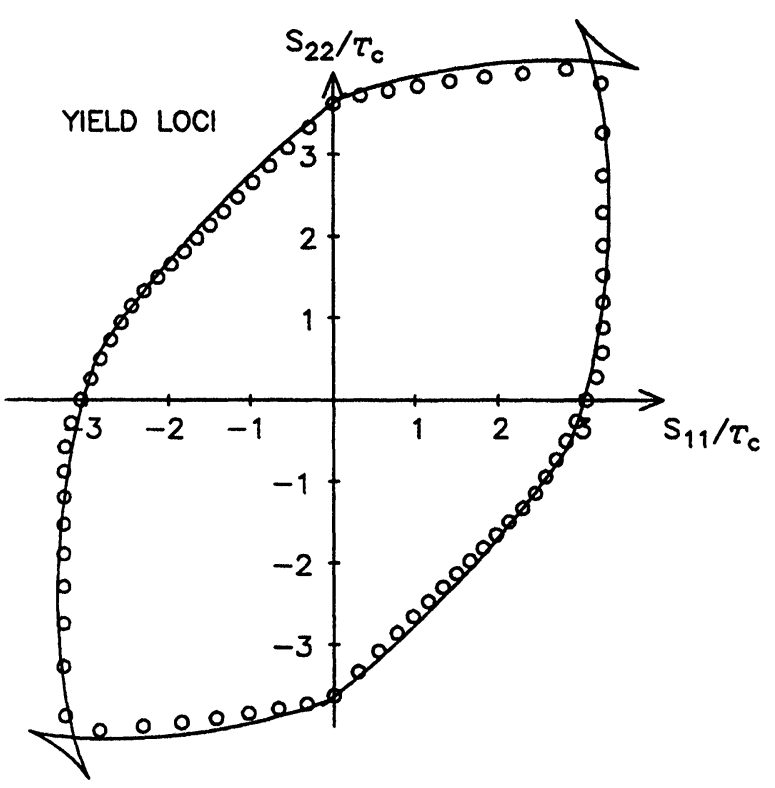

(a)

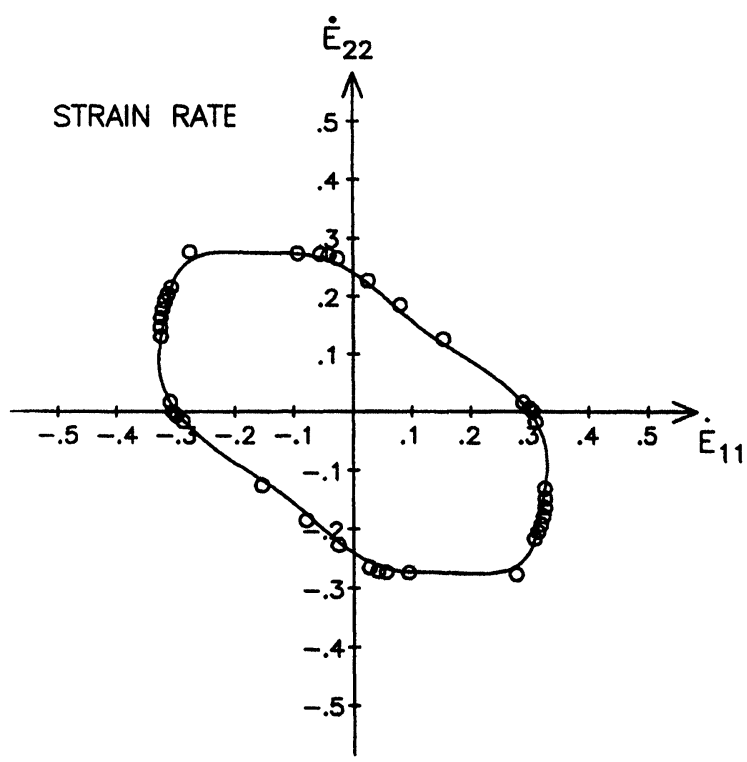

(b)

Figure 1 Equipotential surfaces in stress space and strain rate space of the Brass single component texture, $\{011\}<211>$. Rate insensitive crystal plasticity (open symbols) is compared with Sextus. a) $S_{11}-S_{22}$ section in stress space and b) $\dot{E}_{11}-\dot{E}_{22}$ section in strain rate space. 


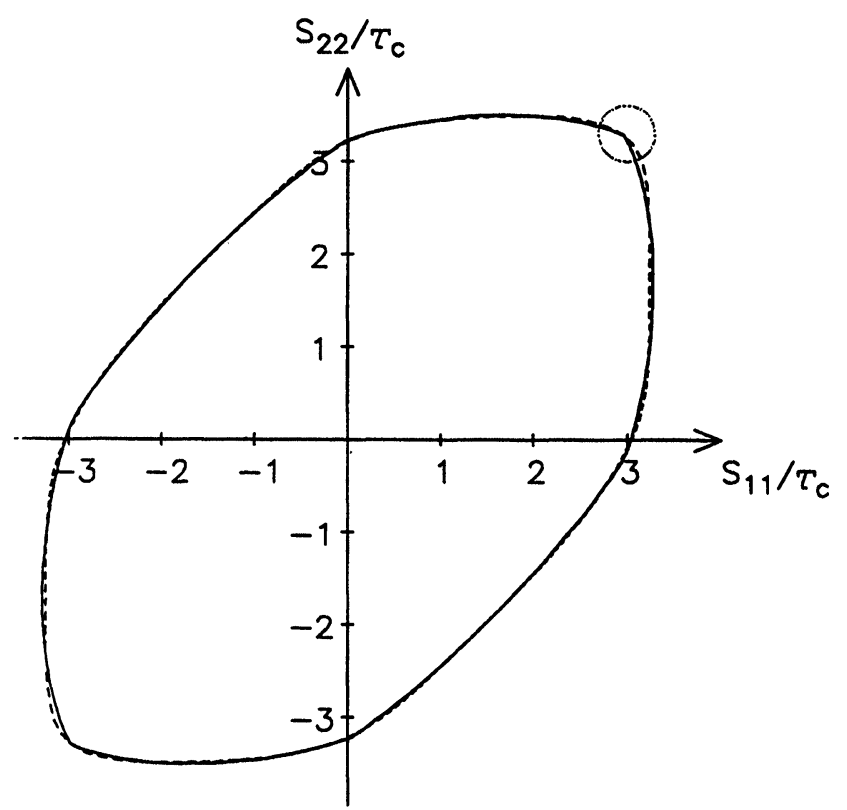

(a)

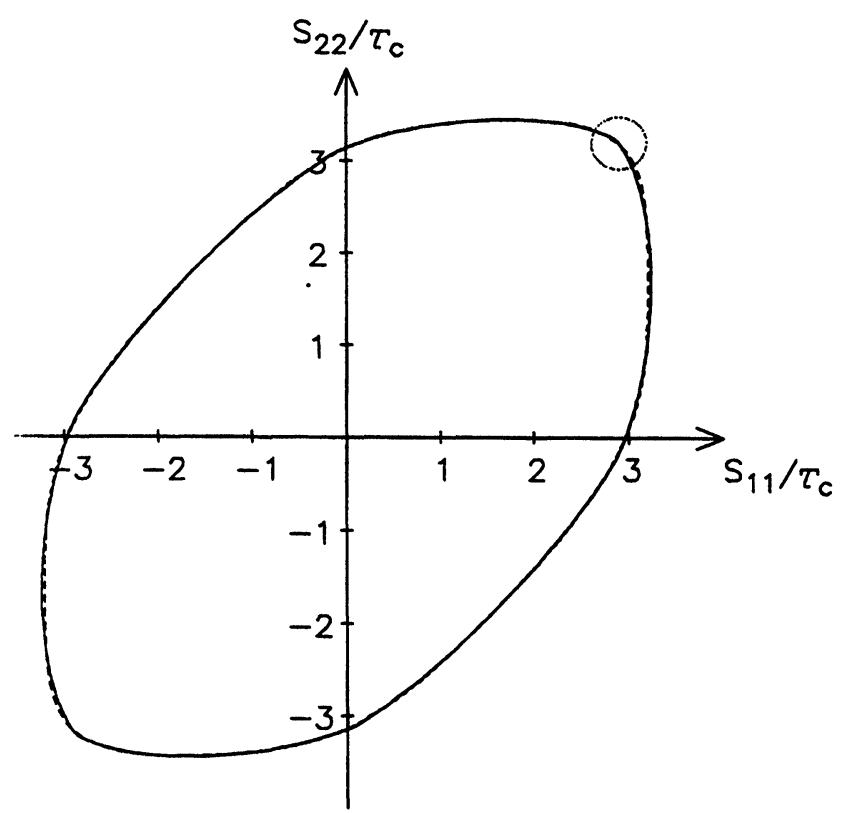

(b)

Figure $2 S_{11}-S_{22}$ sections of the yield loci of the can body stock material. Crystal plasticity (dashed) is compared with Sextus (full line).

a) rate insensitive and $b)$ rate sensitive $(m=.05)$. 


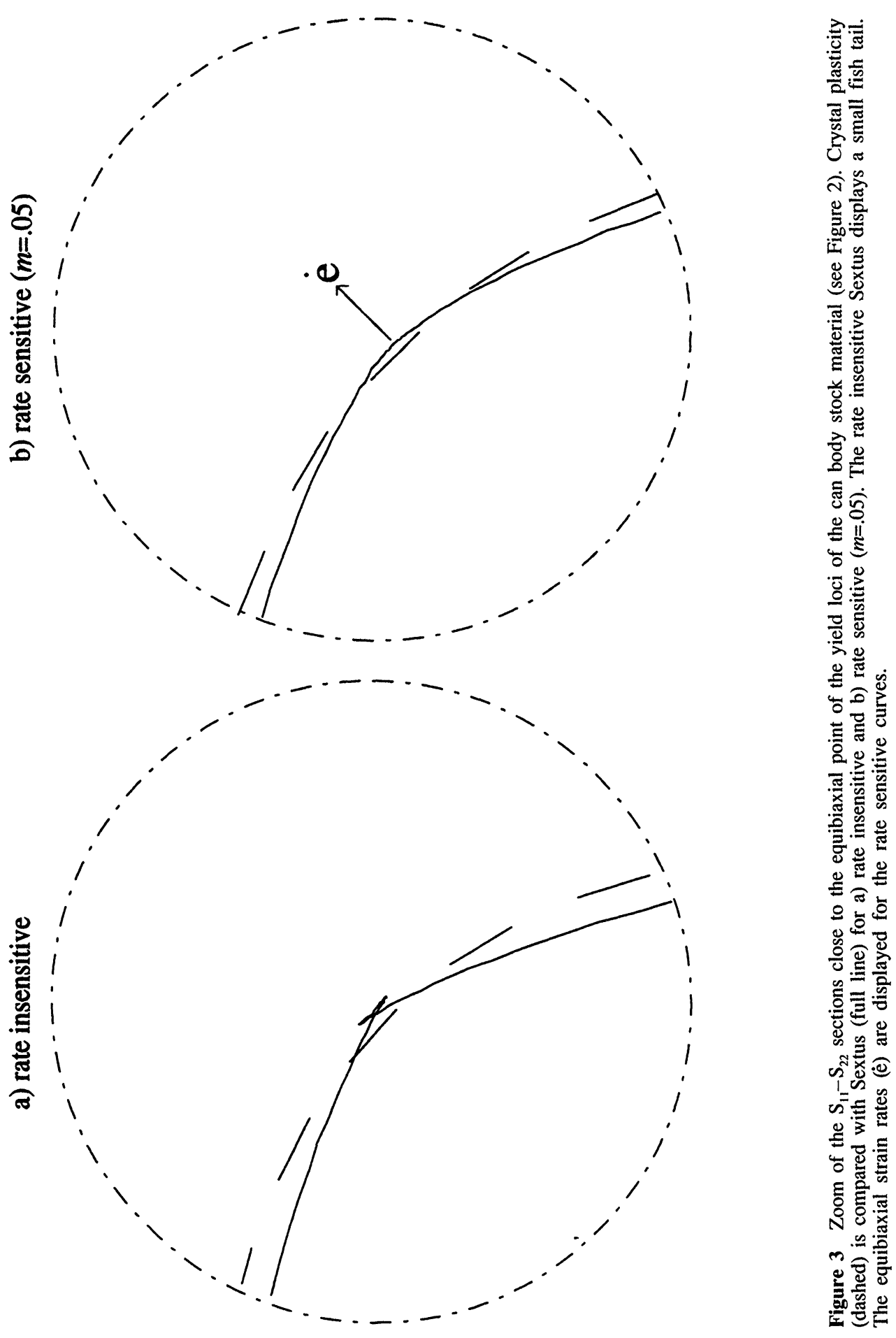


However, sharp textures (as the Brass single component texture introduced above) display fish-tails, even for $m=.05$. Unrealistic high $m$ values are necessary to remove the problem of fish-tails for such textures.

The rate-sensitive approach produces analytical yield surfaces that fall within the envelope of the rate-insensitive ones and are generally more rounded at the equibiaxial corners. Nevertheless, the fit is much better than that obtained using a fourth-order function, as evident in Figure 4.

\section{(iii) Comparison between crystal plasticity and analytical yield surface sections}

Figures 4 and 5 compare yield surface sections $\left(S_{11}-S_{22}\right.$ and $\left.S_{11}-S_{12}\right)$ calculated using the fourth- and sixth-order functions, respectively, with the ones obtained from crystal plasticity for the can body stock material. Quartus (Figure 4) underestimates the crystal plasticity yield surface at equibiaxial and uniaxial positions and overestimates it at planestrain; it provides only an approximation of the crystal plasticity results. Sextus, however, displays much better agreement with crystal plasticity data (Figure 5), although small discrepancies are still seen near equibiaxial and, to a lesser extent, uniaxial stress states ${ }^{2}$. The quality of the fit is also very good for each section involving the other principal stress $\left(S_{22}\right)$ and all other shear stress components $\left(S_{23}\right.$ and $\left.S_{13}\right)$.

The crystal plasticity and analytical $S_{11}-S_{22}$ and $S_{11}-S_{12}$ sections of the automotive alloy are displayed in Figure 6; the fitting is nearly perfect for this case. It is worth mentioning that all these fits shown in Figures 4-6 were obtained from the same rate sensitive $(m=.05) \beta$ coefficients calculated from a single fitting procedure. Only the texture coefficients were changed from one material to another to calculate the corresponding analytical potentials.

\section{(iv) Relative yield strength $\sigma / \sigma(0)$ and $R$-value predictions}

The procedure for calculating the R-value is identical for crystal plasticity and Quartus or Sextus. The contraction ratio $\mathrm{q}$ that gives the minimum plastic work rate (strain rate potential) must be found for specified angular positions of the sample with respect to the rolling direction $(\mathrm{RD})$ :

$$
\frac{\dot{\mathrm{W}}(\mathrm{D})}{\tau}=\psi(\mathbf{D})=\min
$$

with:

$$
D^{P}(q)=\left(\begin{array}{ccc}
1 & 0 & 0 \\
0 & -q & 0 \\
0 & 0 & -1+q
\end{array}\right)
$$

and:

$$
D_{i j}^{\theta}=g_{k i}^{\theta} \cdot D_{k l}^{p} \cdot g_{l j}^{\theta}
$$

\footnotetext{
${ }^{2}$ The use of an eighth-order function does not improve the fitting procedure significantly, while its use increases the calculation time and non-convexity problems.
} 


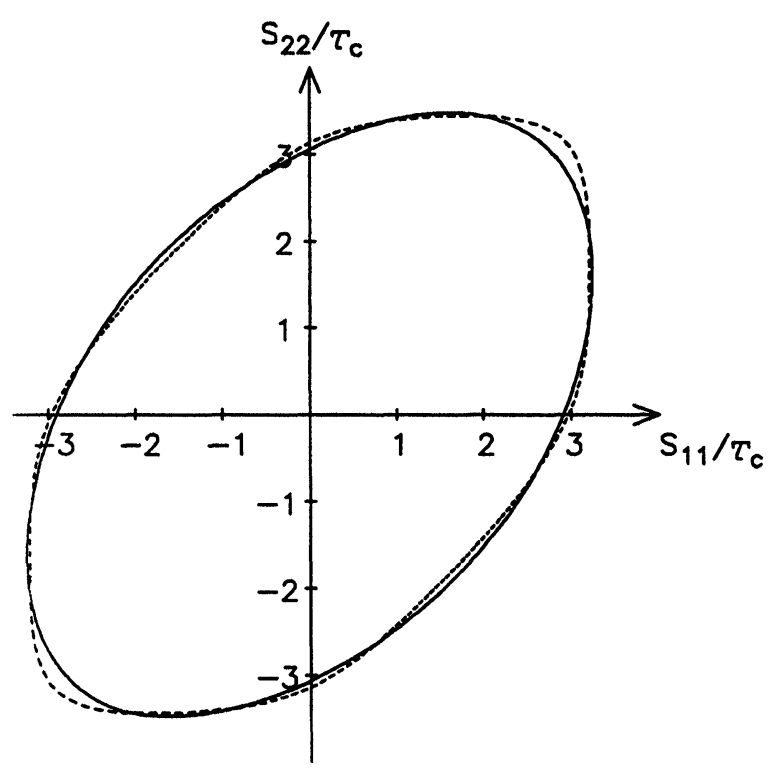

(a)

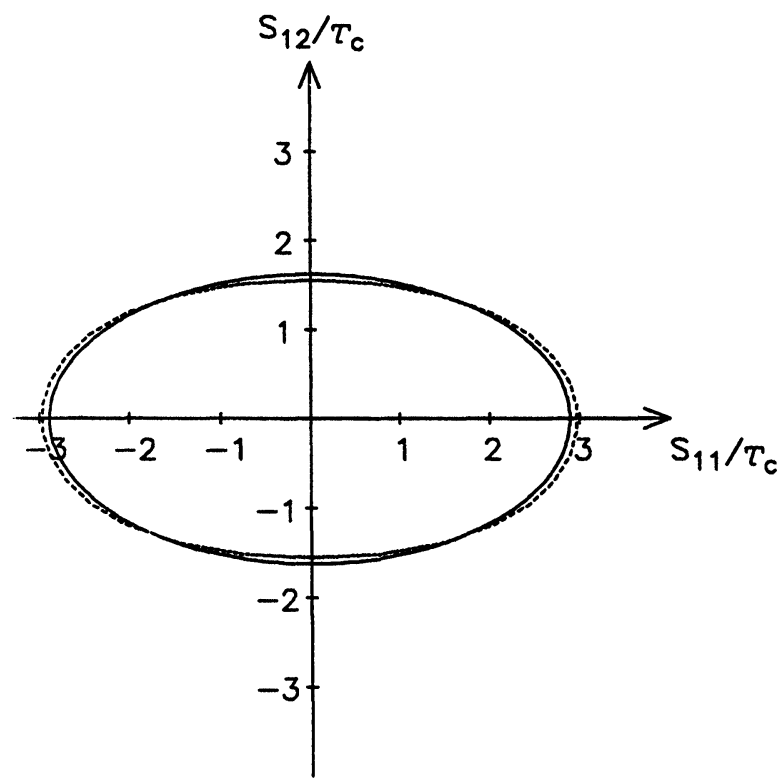

(b)

Figure 4 Rate sensitive $(m=.05)$ equipotential surfaces in stress space (yield loci) of the can body stock material. Crystal plasticity (dashed) is compared with Quartus (full line).

a) $S_{11}-S_{22}$ section and b) $S_{11}-S_{12}$ section. 


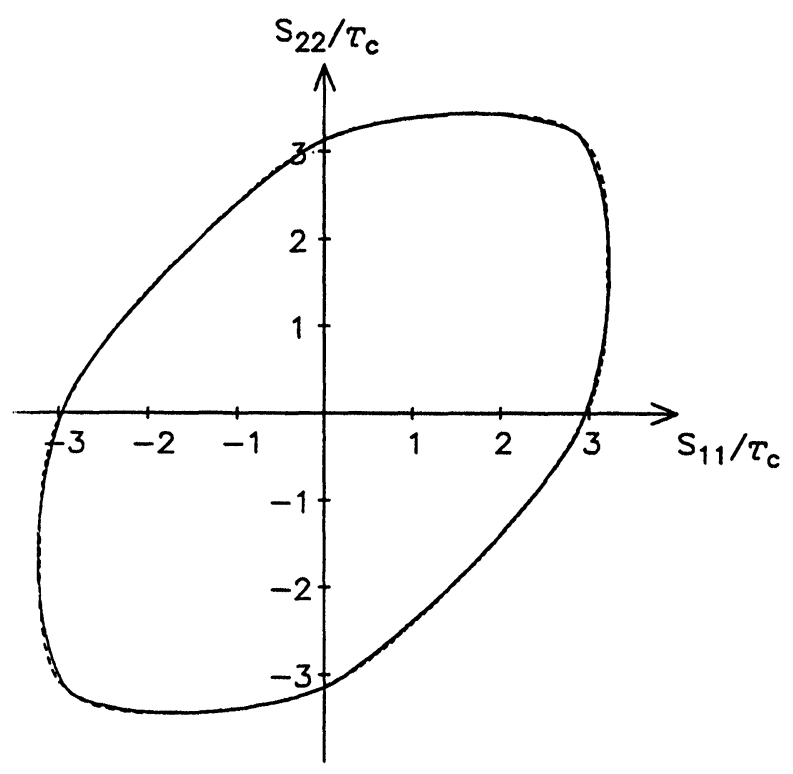

(a)

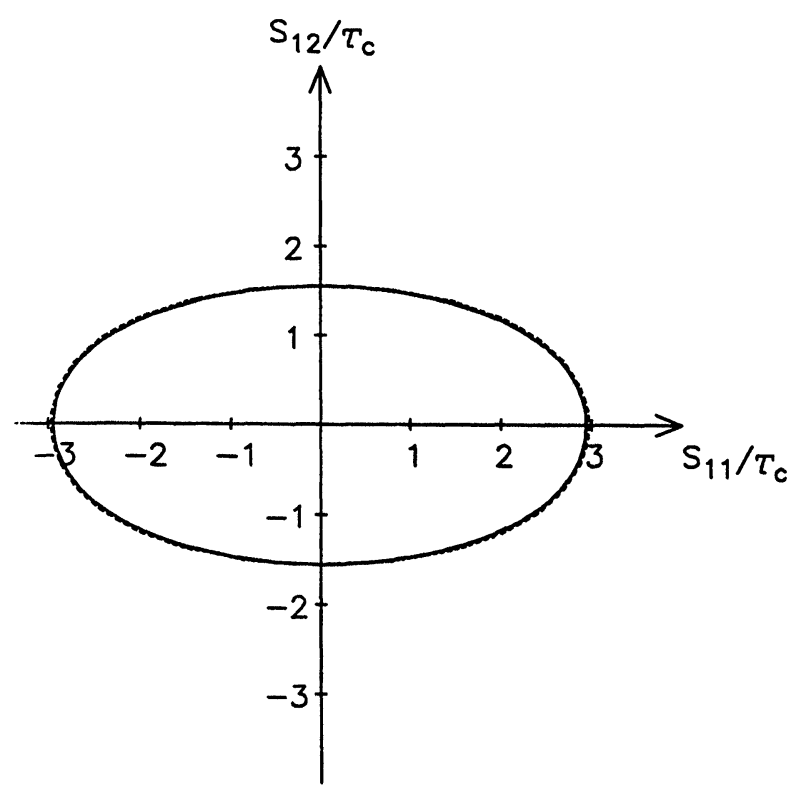

(b)

Figure 5 Rate sensitive ( $m=.05$ ) equipotential surfaces in stress space (yield loci) of the can body stock material. Crystal plasticity (dashed) is compared with Sextus (full line).

a) $S_{11}-S_{22}$ section and b) $S_{11}-S_{12}$ section. 


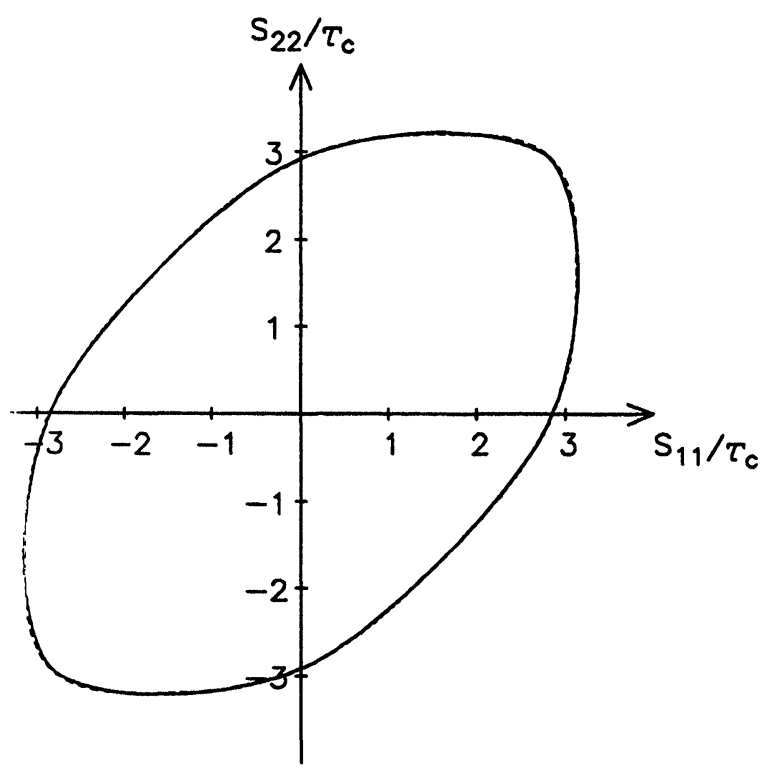

(a)

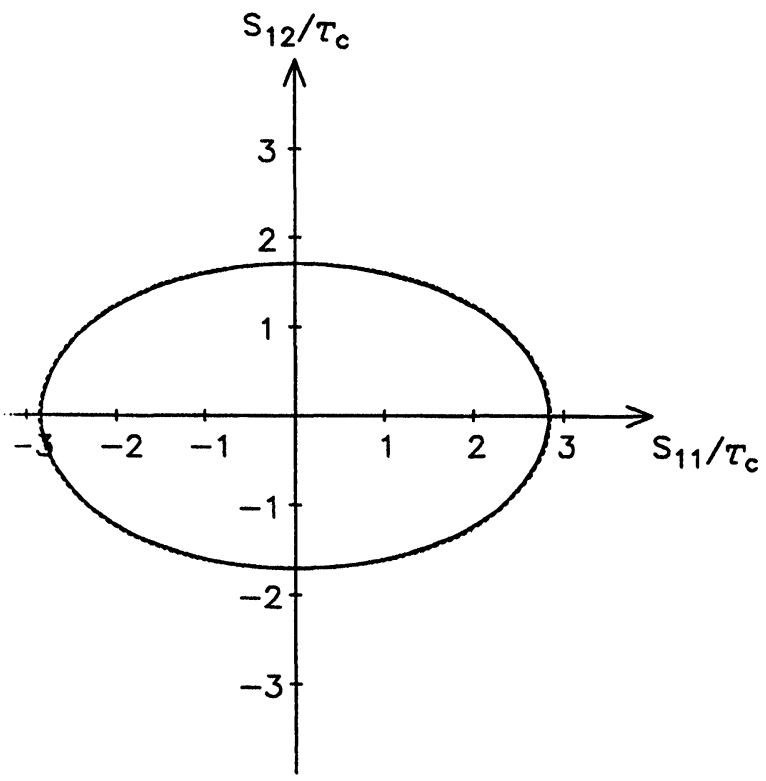

(b)

Figure 6 Rate sensitive ( $m=.05$ ) equipotential surfaces in stress space (yield loci) of the automotive material. Crystal plasticity (dashed) is compared with Sextus (full line).

a) $S_{11}-S_{22}$ section and b) $S_{11}-S_{12}$ section. 
where $\mathbf{D}^{\mathrm{P}}$ represents the strain rate tensor in principal strain (along $R D$ ), and $\mathbf{D}^{\theta}$ the rotated strain rate tensor of an angle $\theta$ about the normal direction between the rolling and the transverse direction. $\mathbf{g}^{\theta}$ is the corresponding rotation matrix. Once the contraction ratio $\mathrm{q}_{\min }^{\theta}$ that gives the minimum plastic work rate has been determined for a selected angular position $\theta$, the $R$-value is given using the following relationship:

$$
R(\theta)=\frac{q_{\min }^{\theta}}{1-q_{\min }^{\theta}}
$$

The relative yield strength (with respect to the one along $\mathrm{RD}$ ) is obtained from the normalized macroscopic plastic work rate that corresponds to that minimum:

$$
\frac{\sigma(\theta)}{\sigma(\mathrm{RD})}=\frac{\dot{\mathrm{W}}\left(\mathrm{q}_{\min }^{\theta}\right)}{\dot{\mathrm{W}}\left(\mathrm{q}_{\min }^{\mathrm{RD}}\right)}
$$

Figures 7 and 8 illustrate yield stress ratios and R-value predictions, respectively, for the canning and automotive alloys introduced above. These figures show calculations from crystal plasticity and the fourth- and sixth-order analytical functions. A rate sensitive $(m=0.05)$ full constraint model was used.

The analytical $\sigma / \sigma_{(0)}$ curves (Figure 7) show almost no sensitivity to the chosen order of the function. Values obtained from Quartus and Sextus fit relatively well the crystal plasticity curves. The analytical R-values (Figure 8) are, however, much more sensitive to the order of the function. While Quartus underestimates the anisotropy of the crystal plasticity predictions, Sextus overestimates this anisotropy, especially for the can body stock material. For materials displaying little anisotropy, Quartus can fit as well as Sextus the crystal plasticity R-values, although the general yield surface shapes of Quartus are not satisfactory.

While the stresses calculated from high order analytical functions are close to the ones calculated from crystal plasticity, their slopes (which define the strain rates and therefore the q-values) can differ significantly. This is particularly the case at the portion of the yield surface where the R-value is calculated (at $S_{22}=0$, for all shear terms $S_{i j}=0$ ). Only a small change in the strain rate (or in the $q$ value) can induce a much larger change in the R-value, see Equation (19). This explains why the R-values predicted by Sextus do not fit crystal plasticity as well as expected.

\section{CONCLUSIONS}

1- The sixth-order inverse potential function provides a better tool than the fourthorder function (despite its non-convexity problems) to fit the inverse potential and yield surface shapes predicted from crystal plasticity. This function is therefore able to "predict" adequately the strain rate gradients in sheet forming operation. This function is currently being implemented into a finite element code, providing a yield surface prediction very close to the one obtained directly from crystal plasticity, but with only a fraction of the calculation time. The advantage of such a method lies in the possibility to take easily into account the texture evolution during the simulation by simply update the analytical function using the new texture coefficients, without performing a new lengthily fitting procedure. 


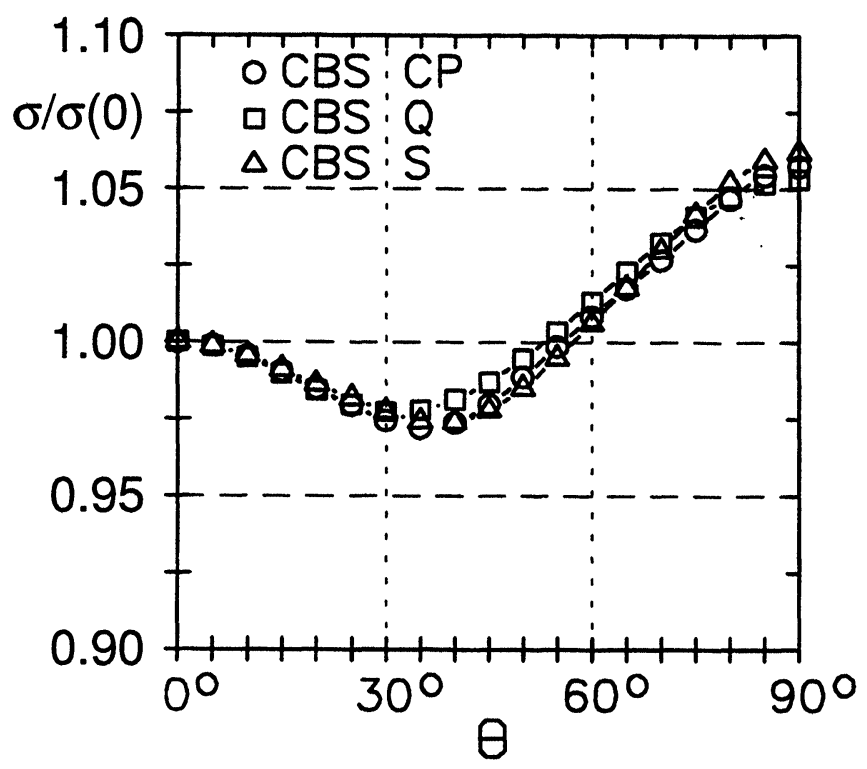

(a)

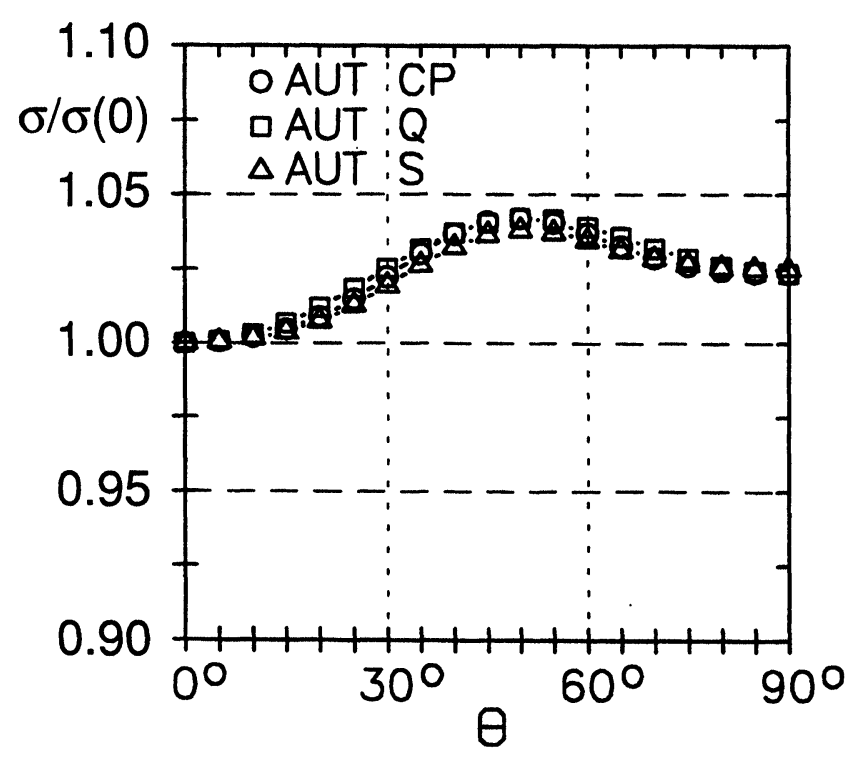

(b)

Figure 7 Rate sensitive ( $m=.05)$ crystal plasticity (CP), Quartus (Q) and Sextus (S) angular variation of $\sigma / \sigma(0)$ for a) can body stock (CBS) and b) automotive (AUT) material. 


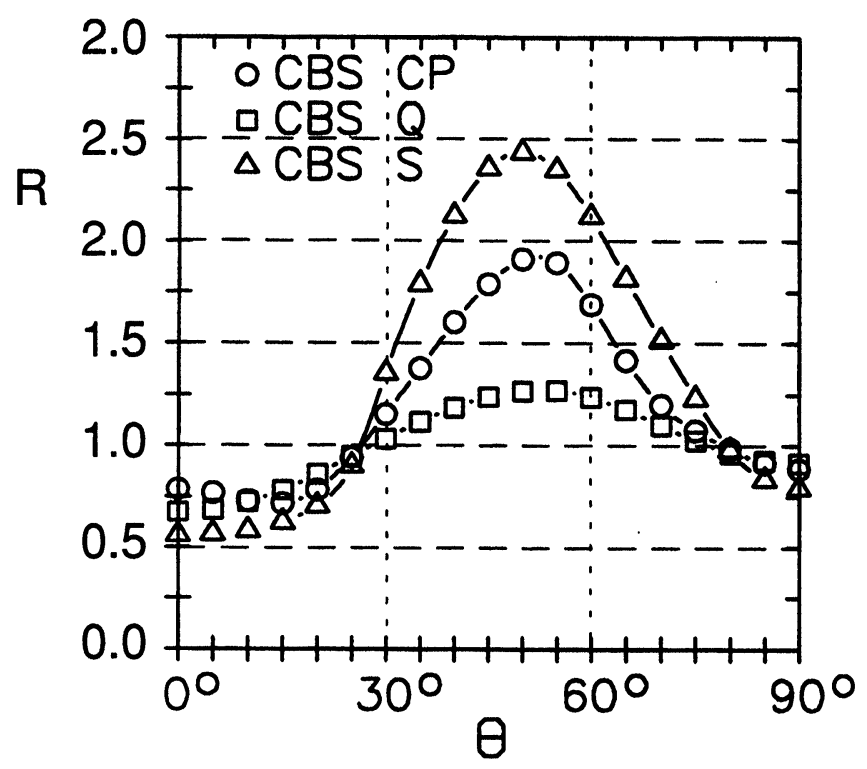

(a)

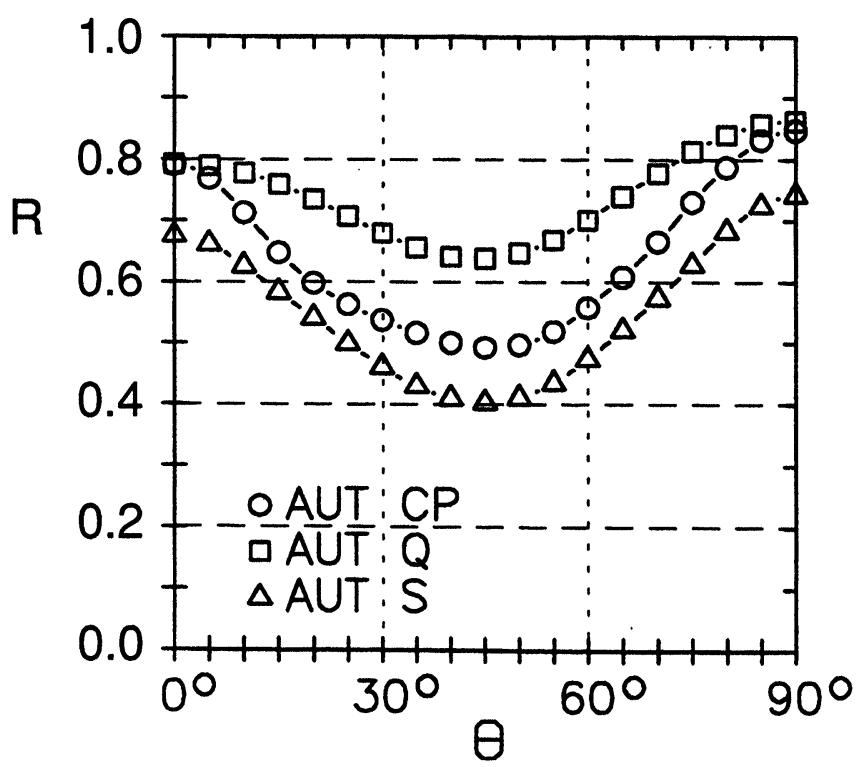

(b)

Figure 8 Rate sensitive ( $m=.05$ ) crystal plasticity (CP), Quartus (Q) and Sextus (S) angular variation of $\mathrm{R}$ for a) can body stock (CBS) and b) automotive (AUT) material. 
2- However, this analytical function can "predict" with much less accuracy the Rvalues. The anisotropy is generally overestimated due to the extreme sensitivity of $R$ with the slope of the yield surface at the tensile direction. This should affect the earing predictions. Results of finite element analysis and comparisons with experiments will be published in a future paper.

\section{Acknowledgements}

The authors wish to thank Brigitte Bacroix for numerous and helpful discussions and Prof. Günther Gottstein for making possible the realization of a part of this work at the Institut für Metallkunde und Metallphysik, Aachen (Germany).

\section{References}

Arminjon, M. and Bacroix, B. (1991). Acta Mech. 88, pp. 219-243.

Arminjon. M., Bacroix, B., Imbault. D. and Raphanel, J. L. (1994). Acta Mech. 107, pp 33-51.

Asaro, R. J. and Needleman, A. (1985). Acta Metall. 33, pp. 923-953.

Bacroix, B. and Gilormini, P. (1995). Modelling Simul. Mater. Sci. Eng. 3, pp. 1-21.

Barlat, F. and Lian, J. (1989). Int. J. Plasticity 5, pp. 51-66.

Barlat, F., Chung, K. and Richmond, O. (1993). Int. J. Plasticity 9, pp. 51-63.

Beaudoin, A. J., Dawson, P. R., Mahur, K. K., Kocks, U. F. and Korzekwa, D. A. (1994). Comp. Meth. in Appl. Mech. and Eng. 117, pp. 49-70.

Fletcher. (1970). Comp. J. 13, p. 317.

Hill, R. (1948). Proc. R. Soc. A 193, pp. 281-297.

Hill, R. (1979). Math. Proc. Camb. Phil. Soc. 85, pp. 179-191.

Hill, R. (1987). J. Mech. Phys. Solids 35, pp. 22-33.

Hill, R. (1993). Int. J. Mech. Sci. 35, pp 19-25.

Kocks, U. F. and Canova, G. R. (1981). Deformation of Polycrystals, Ris $\varnothing$ National Laboratory, Roskilde, Denmark.

Toth, L. S., Gilormini, P. and Jonas, J. J. (1988). Acta Metall. 36, pp. 3077-3091.

Van Houtte, P. (1988). Textures and Microstructures 8\&9, pp. 313-350.

Van Houtte, P., Mols, K., Van Bael, A. and Aernoudt, E. (1989). Textures and Microstructures 11, pp. 23-39.

Von Mises, R. (1928). Z. angew. Math. Mech. 8, p. 161.

Ziegler, H. (1977). An Introduction to Thermodynamics, Amsterdam: North-Holland. 